\title{
Characterizing the Brown Spot Pathosystem in Late-Harvest Table Grapes (Vitis vinifera L.) in the California Central Valley
}

Cassandra L. Swett, Department of Plant Science and Landscape Architecture, University of Maryland, College Park 201742; and Tyler

Bourret and W. Douglas Gubler, Department of Plant Pathology, University of California, Davis 95006

\begin{abstract}
Swett, C. L., Bourret, T., and Gubler, W. D. 2016. Characterizing the brown spot pathosystem in late-harvest table grapes (Vitis vinifera L.) in the California central valley. Plant Dis. 100:2204-2210.

Brown spot, caused by Cladosporium spp., is becoming a problematic postharvest disease of late season table grape (Vitis vinifera) in the California central valley, and management is hindered by knowledge gaps in disease etiology and epidemiology. Brown spot is herein described as a pre- and postharvest dry rot typified by an external brown to black spot or black mycelium which encases the placenta. Isolates in the Cladosporium herbarum and $C$. cladosporioides species complexes were recovered from 85 and $5 \%$ of brown-spot affected berries, respectively. Five isolates in the $C$. herbarum species complex, representing three phylogenetically distinct species

(C. limoniforme, C. ramotenellum, and C. tenellum), and one C. cladosporioides isolate all caused brown spot symptoms under cold-storage conditions, with and without mechanical wounding. Isolate virulence was similar $(P>$ 0.05 ) based on disease incidence and severity on intact berries but severity varied on wounded berries $(P<0.001)$. Surface disinfestation reduced severity of cluster rot development following 2 weeks in cold storage $(P=0.027)$ but incidence was not affected $(P=0.17)$. This work provides foundational information on brown spot pathosystem etiology and biology in late-harvest table grape, which can be used to improve management.
\end{abstract}

Table grape (Vitis vinifera L.) production in the central valley represents the greatest portion of the grape market in California, due predominately to the large profit margins in export and late-season (winter holiday) markets. Postharvest disease losses during transport and long-term storage have become increasingly problematic as producers seek to extend storage durations prior to and during transport (up to 6 months) (Fernández-Trujillo et al. 2008; Rooney-Latham et al. 2008). Brown spot, a disease caused by Cladosporium spp., contributes to postharvest losses, primarily in late-harvest table grapes. In the central valley, this disease is emerging as a major driver of fruit damage during cold storage and transport, resulting in costly reimbursable claims on exported fruit and rendering fruit unmarketable for high-value winter market sale. Increasing brown-spot-associated losses may be a product of alterations in viticulture practices, storage methodologies, differences in susceptibility of currently grown cultivars, or the emergence of additional Cladosporium spp.

Brown spot has been described as both a field rot (preharvest) disease in wine, table, and juice grape (Briceño and Latorre 2008; Ouchi et al. 1976; Sprague 1952) and a postharvest spoilage problem in table grape (Harvey and Pentzer 1960). Early studies characterized the disease on early-harvest table grape, primarily 'Emperor' and 'Flame Tokay' (both no longer produced) in California in the 1950s and 1960s, where it was called brown spot (Delp et al. 1951; Harvey and Pentzer 1960). The disease is characterized by a circular, firm, dark brown to black spot, usually at the blossom end or localized to one side of the berry (Harvey and Pentzer 1960; Ouchi et al. 1976). Spot formation is followed by withering of the berry and sporulation on the fruit surface (Harvey and Pentzer 1960). In some cases, the definition of brown spot is expanded to include green fungal growth which develops on otherwise healthy green berries in the field (alternately referred to as green-berry), resulting in small black spots on the berry surface (Briceño and Latorre 2007, 2008; Latorre et al. 2011). In all descriptions, the disease is characterized as a case of

Corresponding author: C. L. Swett; E-mail: clswett@umd.edu

Accepted for publication 9 June 2016.

http://dx.doi.org/10.1094/PDIS-11-15-1343-RE

(C) 2016 The American Phytopathological Society surface degradation, without internal growth (Briceño and Latorre 2008; Harvey and Pentzer 1960; Ouchi et al. 1976).

In California table grapes, brown spot has not been observed preharvest and it is typically only a minor postharvest problem during storage. However, the disease has recently been found causing major damage on cultivars harvested and stored in the fall; mainly 'Red Globe' but also 'Crimson' and 'Autumn King'. In addition to symptoms described above, several previously undescribed internal symptoms have also been observed in these cultivars. It is unclear whether disease only initiates after harvest or also can develop in the field.

The primary pathogens reported to cause brown spot are Cladosporium cladosporioides and C. herbarum (Briceño and Latorre 2008; Ouchi et al. 1976; Riggs 2006); both of these typify morphospecies and subgeneric clades referred to as species complexes (Bensch et al. 2010, 2012, 2015; Schubert et al. 2007). C. cladosporioides is considered the more virulent of the two, causing more severe symptoms than $C$. herbarum in the absence of wounding (Briceño and Latorre 2008; Ouchi et al. 1976; Riggs 2006). In preliminary surveys in California table grape vineyards, members of both complexes were associated with brown spot symptoms. Although it is assumed that members of the $C$. cladosporioides species complex are the primary drivers of disease, the relative epidemiological importance of different species is not known for this region. Studies to evaluate the roles of these species in driving brown spot can inform future management studies and also lend insight into correspondence between recent phylogenetic revisions and ecological traits (Bensch et al. 2010, 2012; Schubert et al. 2007).

Brown spot management aims to suppress preharvest infections from epiphytic Cladosporium populations established at the time of harvest. Fruit are typically fungicide treated shortly before harvest, then stored between 0 and $-2^{\circ} \mathrm{C}$, at $85 \%$ relative humidity (RH), and fumigated with $\mathrm{SO}_{2}$ (Harvey and Pentzer 1960; Fernández-Trujillo et al. 2008). Reliance on this approach is based on the assumption that disease develops primarily from surface-dwelling propagules that infect fruit while in storage. Although it is highly likely that postharvest infections contribute to disease development, studies are lacking which evaluate the efficacy of reducing epiphytic populations for postharvest control of brown spot. Some studies indicate that Cladosporium spp. may also establish internal infections in the field prior to harvest in a mode similar to Botrytis cinerea (Dugan et al. 2002), in which case, integration of early-season preharvest treatment strategies may be critical to reducing postharvest losses. 
Table 1. Brown spot incidence and severity of infection pre- and postharvest on clusters and in storage boxes, respectively

\begin{tabular}{lcc}
\hline Infection & Preharvest $(\%)^{\mathbf{y}}$ & Postharvest $(\%)^{\mathbf{z}}$ \\
\hline Incidence & $21 \pm 6$ & $88 \pm 3$ \\
Severity & $0.22 \pm 0.06$ & $0.41 \pm 0.04$ \\
\hline
\end{tabular}

y Preharvest disease incidence quantified as the percentage of clusters with at least one diseased berry in each row and disease severity quantified as the percentage of diseased berries per affected cluster, for two assay dates combined ( \pm standard error $[\mathrm{SE}]$ ).

${ }^{\mathrm{z}}$ Postharvest disease incidence rated after 5 weeks in storage as the percentage of boxes with at least one diseased berry in each row and severity evaluated as the percentage of berries per box (out of approximately 800) with symptoms, for two assay dates combined $( \pm \mathrm{SE})$.
The focus of this study was on late-harvest Red Globe in the California central valley. The objectives were to (i) evaluate brown-spotassociated yield losses and disease symptomology, (ii) characterize diversity and virulence of Cladosporium spp. associated with brown spot in cold storage, and (iii) evaluate efficacy of epiphyte-targeted surface treatment strategies for controlling disease development postharvest.

\section{Materials and Methods}

Brown-spot-associated yield losses and symptomology. The incidence and severity of brown spot was evaluated in both field ratings before harvest and in cold storage after harvest, and symptoms observed in ratings were used to develop a description of the disease

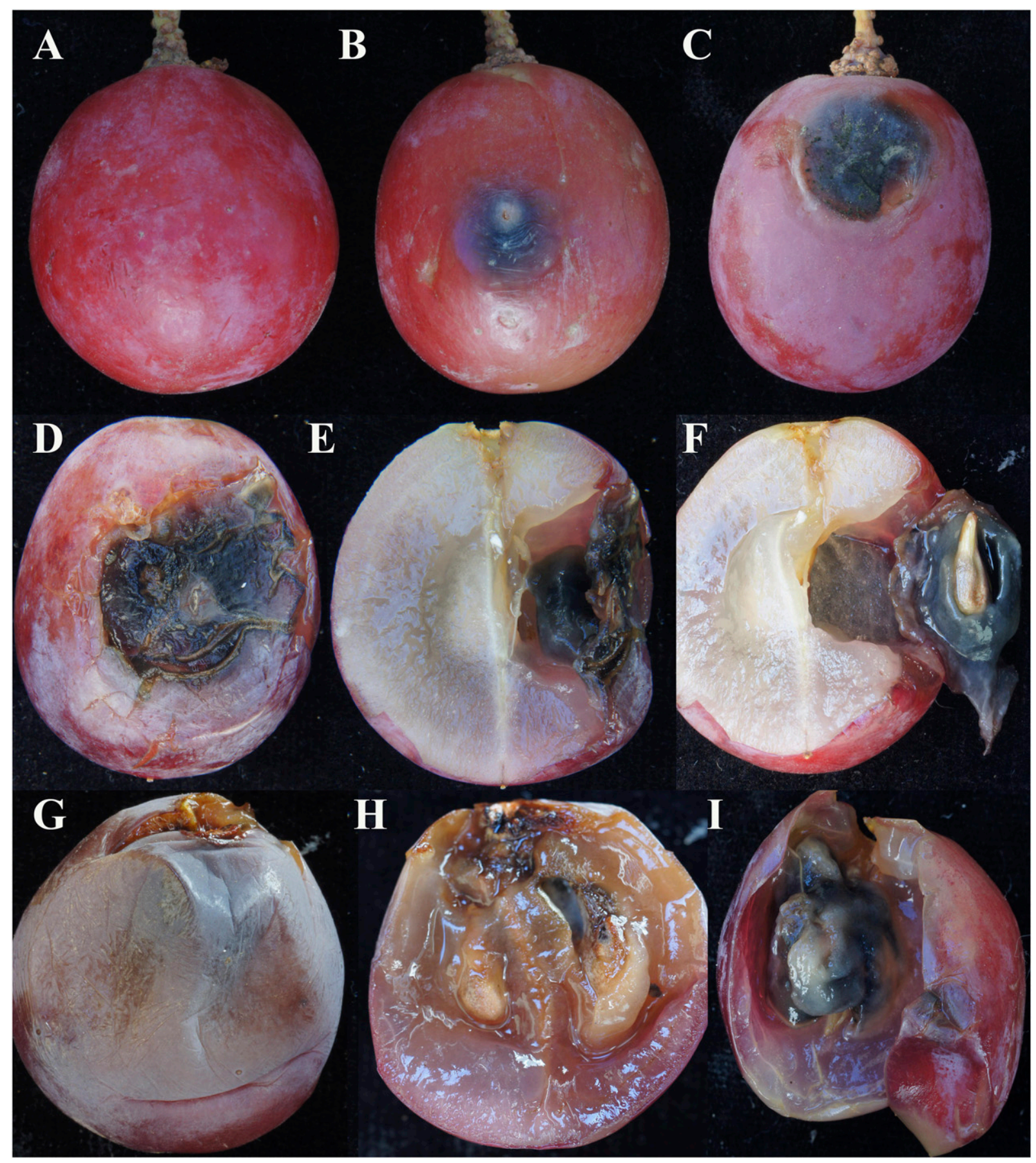

Fig. 1. Brown spot disease symptoms postharvest contrasting A, a healthy berry with B, early brown spot symptom development; $\mathbf{C}$, brown spot with cavity development and fungal sporulation; D, advanced spot symptom with $\mathbf{E}$, internal growth which $\mathbf{F}$, replaced the placenta and encased the seed; $\mathbf{G}$, berry with no external spot symptoms but $\mathbf{H}$, with Cladosporium spores lining the interior of the mycelial mat; and I, internal growth that completely replaced placenta. 
in late-harvest table grape in central California. All collections were conducted in Kern County, CA (GPS: $35^{\circ} 45.470$ N, $119^{\circ} 08.527 \mathrm{~W}$ ).

Preharvest brown spot incidence and severity was rated in the field during the harvest period, on 11 September 2013 and again on 1 October 2013. Three clusters were collected from each of three rows, and all berries were examined for brown spot symptoms (brown to black spot) and signs of Cladosporium spp. (olive-green sporulation) both externally and internally. All symptomatic berries were surface disinfested by washing in $0.1 \%$ Tween 20 , followed by immersion in $70 \%$ ethanol for $30 \mathrm{~s}$ and $0.1 \%$ sodium hypochloride $(\mathrm{NaClO})$ for $3 \mathrm{~min}$. The affected region (spot or internal growth) was aseptically excised and placed on petri dishes containing Rose Bengal agar (Difco, Bacto). Emerging colonies were identified as Cladosporium spp. based on culture characteristics and conidia morphology (Barnett and Hunter 1998). Disease incidence was quantified as the proportion of clusters with at least one symptomatic berry, and severity was analyzed conditionally as the estimated proportion of the cluster affected (McRoberts et al. 2003). Harvest dates were combined in both incidence and severity calculations if they were not significantly different.

Postharvest disease development was evaluated 6 weeks after harvest for five boxes in each of eight rows, in which each box contained approximately 800 berries ( 10 bags with 80 berries each). Ratings were conducted twice, for berries harvested on 1 October 2013 and berries harvested on 6 November 2013. Berries were stored under commercial storage conditions $\left(0\right.$ to $-2^{\circ} \mathrm{C}, 85 \% \mathrm{RH}$, with daily $\left.\mathrm{SO}_{2}\right)$ for 6 weeks, and then brown spot was rated as the proportion of berries per box with brown spot symptoms (assuming 800 berries per box on average). A subset of symptomatic berries was surface disinfested and emerging colonies were identified as Cladosporium spp., as above. Disease incidence was analyzed as the proportion of boxes per row with at least one symptomatic berry, averaged across the eight rows. Disease severity was analyzed as the average proportion of symptomatic berries per box in each row, in those boxes with disease, averaged for all rows. As above, harvest dates were combined in both incidence and severity calculations if they were not significantly different.

To characterize the breadth of brown spot symptoms observed in the study, all symptoms were collected and photographed, and Cladosporium spp. association was evaluated based on (i) micro- and macrocondial morphologies typical of the genus (Barnett and Hunter 1998, Bensch et al. 2012) and (ii) isolation of Cladosporium spp. from a representative subset of berries postharvest (about 10\%). For the latter, berries were surface disinfested by washing in $0.1 \%$ Tween 20 and soaking for $30 \mathrm{~s}$ in $70 \%$ ethanol and then $3 \mathrm{~min}$ in $0.1 \% \mathrm{NaClO}$, and symptomatic regions were cut into approximately $1-\mathrm{cm}^{2}$ segments and placed on Rose Bengal agar. Identity as Cladosporium spp. was confirmed based on conidia morphology, as above.

Diversity and virulence of Cladosporium spp. associated with brown spot in cold storage. Species diversity and relative abundance were evaluated in 10 symptomatic berries harvested between 1 October and 6 November 2013 and placed in cold storage (maintained at between 1 and $4^{\circ} \mathrm{C}$ ). Surveys were conducted on 18 December 2013 and again on 5 February 2014. Berries were surface disinfested as described above, and the symptomatic region was aseptically segmented into four $5-\mathrm{mm}^{2}$ segments and placed on $100-\mathrm{mm}$ petri dishes containing Rose Bengal agar.

Each colony emerging from each segment was pure cultured as a single germinating hyphal tip. Genomic DNA was extracted directly from mycelium of 10 isolates using the Invitrogen PrepMan DNA extraction kit (following the PrepMan protocol for microbial DNA extraction). A portion of the actin gene containing two conserved introns was amplified with ACT-512F and ACT-783R primers and cycling conditions previously described (Carbone and Kohn 1999) using GoTaq Green MM (Promega Corp.). Polymerase chain reaction (PCR) products were purified using the MoBio PCR purification kit before sequencing. Raw data obtained from ABI 3730xl sequencers was curated using Chromaseq (Maddison and Maddison 2014), phred (Green and Ewing 2015), and phrap (Green 2015).

Sequences from grape berry isolates were compared with actin sequences obtained from voucher specimens using BLAST searches (Altschul et al. 1990) and phylogenetic inference methods. Sequences obtained from GenBank and the 10 sequences obtained in the current study were aligned using mafft-linsi (Katoh and Standley 2013); the alignment was trimmed with AliView (Larsson 2014), resulting in a 146-by-214-bp matrix. This alignment was filtered using the least stringent options available using the Gblocks server (Castresana 2000), trimming from 214 to $137 \mathrm{bp}$. MEGA7 (Kumar et al. 2016) was used to produce maximum-parsimony and neighbor-joining trees. The parsimony tree was obtained using heuristic maximum-parsimony SPR searches and neighbor joining using maximum composite likelihood with $\gamma$-distributed rates; both tree searches used partial deletion with a 95\% cutoff which included all 137 sites. The AICc criterion indicated the use of the TPM3uf $+\mathrm{I}+\mathrm{G}$ nucleotide substitution model using jModelTest 2 (Darriba et al. 2012). Garli 2.01 (Zwickl 2006) was used to infer the most likely tree using 200 searches with default values and genthreshfortopoterm $=25,000 ; 10,000$ was used for bootstrap iterations.

Virulence in cold storage $\left(4^{\circ} \mathrm{C}, 100 \% \mathrm{RH}\right)$ was evaluated for five isolates in the $C$. herbarum species complex (two C. ramontellum isolates, two $C$. limoniforme isolates, and one $C$. tenellum isolate) and one C. cladosporioides isolate. Two inoculation treatments were implemented: intact berry inoculation (no wounding) and inoculation on a 1-mm wound. Three berries (subreplicates) were allocated to each inoculation treatment in each of the three replicate incubators for a total of nine berries per isolate per inoculation treatment. In addition, each incubator contained a noninoculated intact fruit and noninoculated wounded fruit treatment (three berries per treatment) to serve as negative controls.

In preparation for inoculations, Red Globe berries were surface disinfested by washing in $0.1 \%$ Tween 20 and soaking in $70 \% \mathrm{ETOH}$ for $30 \mathrm{~s}$ and $0.1 \% \mathrm{NaClO}$ for $4 \mathrm{~min}$, then dried in a flow hood and allowed to warm to ambient temperature to reduce development of surface condensation as a result of warming from cold-storage conditions. Inoculations were conducted by first suspending spores of cultures grown for 5 to 7 days on potato dextrose agar under ambient light at $27^{\circ} \mathrm{C}$. Cultures were flooded with a sterile solution of $0.5 \%$ $\mathrm{KCl}+0.1 \%$ Tween 20 , spores were dislodged with a sterilized glass rod and poured into a sterile $50-\mathrm{ml}$ falcon tube, and spore concentration counted using a hemacytometer and adjusted to $10^{7}$ spores $/ \mathrm{ml}$. Inoculum was stored at $4^{\circ} \mathrm{C}$ and used within $24 \mathrm{~h}$.

To inoculate all berries, a 5-mm ring of Vaseline was first made on the shoulder of the berry around the inoculation site to prevent the inoculum from rolling off the berry surface. Intact berries were then inoculated by placing a $10-\mu 1$ droplet of $10^{7}$ spores $/ \mathrm{ml}$ at the inoculation site. To wound inoculate, a sterile hypodermic needle was first used to create a 1-mm wound which penetrated just through the epidermis, and the inoculum droplet was placed at the wound site. Berries were placed in plastic incubators on plastic racks (sterilized with $0.5 \% \mathrm{NaClO}$ ). Approximately $3 \mathrm{~cm}$ of sterile water was added to the bottom on each chamber to achieve approximately $97 \% \mathrm{RH}$ and incubators were maintained at $4^{\circ} \mathrm{C}$.

Disease development was evaluated at 2 and 4 weeks postinoculation as the presence or absence of symptoms on each berry. At 4 weeks postinoculation, disease severity was quantified based on the spot diameter on each berry. At this time, the symptomatic region was aseptically excised and placed on Rose Bengal agar, and the emerging Cladosporium colonies from each berry were cultured and examined by microscopy for morphological features consistent with the inoculated isolate. This test was conducted twice.

Analysis of variance (ANOVA) and Tukey's pairwise means comparisons, as implemented in R 2.15.2, were used to asses treatment effects on disease incidence and severity. Percentage data were subjected to an arcsine square-root transformation prior to analysis. Unless otherwise noted, experiment $\times$ treatment interactions were not significant; therefore, data were pooled across experiments to test the significance of main effects.

Efficacy of epiphyte-targeted surface treatment on postharvest disease development. The effect of epiphyte-targeted treatment was evaluated in Red Globe clusters harvested in the fall which were either surface disinfested or untreated. The experiment was arranged in a complete block design, with four blocks (cases) per treatment, four 
clusters per block, and 8 to 27 berries/cluster. The experiment was conducted twice.

Red globe table grape were harvested on 23 October 2013 (experiment one) and 6 November 2013 (experiment two), stored at $4^{\circ} \mathrm{C}$, and treated within 1 week of harvest. Each cluster was thinned to maximize efficacy of disinfestation. Clusters were surface disinfested by rinsing in tap water, briefly submerging in $0.1 \%$ Tween 20 , then soaking in $70 \%$ ethanol for $30 \mathrm{~s}$ and $0.1 \% \mathrm{NaClO}$ for $3 \mathrm{~min}$. This treatment was not intended to fully eradicate Cladosporium populations from the fruit surface but, instead, to mimic effects of an effective fungicide in reducing epiphytic populations. $\mathrm{NaClO}$ is not used for brown spot management but was selected due to ambiguities in the efficacy of commercially available fungicides at the time of the study (C. L. Swett and W. D. Gubler, unpublished data). To control for effects of wetting berries, nondisinfested clusters were gently sprayed with sterile water to sufficiently moisten berries without washing off surface material.

Clusters were air dried in a laminar flow hood for $1 \mathrm{~h}$, rotating to dry the whole cluster. Each cluster was placed in a separate plastic bag and four clusters per treatment were randomly allocated to each of four foam cases (blocks) used by commercial operations, and stored at $4^{\circ} \mathrm{C}$ as described above. The percentage of berries per cluster with brown spot symptoms was evaluated at 1 and 2 weeks after treatment. Emergence of other fruit rot fungi (B. cinerea, Aspergillus spp., and Penicillium spp.) was also recorded, and any ambiguous symptoms (possible co-infections or other diseases) were excluded from analyses.

\section{Results}

Brown-spot-associated yield losses and symptomology. In the field, brown spot was observed on an average of $21 \%$ of clusters, affecting $0.22 \%$ of the berries ( 1 to 2 berries) in the cluster (Table 1 ). Symptomatic berries were typically those in the center of the cluster and were not detectable unless the cluster was dissected. Five weeks after harvest, $88 \%$ of boxes had diseased berries, in which an average of $0.41 \%$ of berries (about 3 of 800 berries) in each box had brown spot symptoms (Table 1).

Brown spot included both external and internal symptoms and signs. The most common symptom was a small- to medium-sized hardened brown spot on the surface of the berry (typically one spot on each berry; Fig. 1). This spot was a product of dark-brown to black hyphae growing below or on the surface of the epidermis, combined with necrosis of the berry tissue. As the spot increased in size, the affected area became sunken, forming a cavity inside the berry, within which the fungus formed a dry black mycelial mat (Fig. 1). This mat often appeared to form within the placenta, encasing the seed, and sporulation on the surface of the mycelial growth was commonly observed (Fig. 1). Less often, disease symptoms and signs manifested as skin darkening, with an internal hollow region containing a dark mycelial mat but without any distinct external spot (Fig. 1). When pulled away from the seed, dark, olive-green spores were commonly observed lining the interior of the mycelial mat (Fig. 1).

Diversity and virulence of Cladosporium spp. associated with brown spot in cold storage. Cladosporium spp. were recovered from $90 \pm 5 \%$ of berries with brown spot symptoms; Alternaria spp. emerged from $5 \pm 2.5 \%$ of berries, and $5 \pm 2.5 \%$ of berries were compromised by contaminants, preventing identification of emerging colonies. Based on BLAST search and phylogenetic analyses, isolates segregated into three species within the $C$. herbarum species complex (C. limoniforme, $C$. ramotenellum, and $C$. tenellum) and one species (C. cladosporioides) within the $C$. cladosporioides species complex (Table 2; Fig. 2). C. herbarum species complex isolates were recovered from $85 \pm 2.5 \%$ of berries; $C$. cladosporioides was recovered from $5 \pm 2.5 \%$ of berries (a single recovery) where it co-occurred with a $C$. herbarum species complex isolate.

Brown spot incidence was evaluated at 2 and 4 weeks postinoculation. There was no effect of isolate $(P=0.39$ at 2 weeks and $P=0.54$ at 4 weeks) and no significant isolate $\times$ inoculation method interaction ( $P=0.1$ at 2 weeks and $P=0.5$ at 4 weeks); therefore, isolates were combined to evaluate effects of inoculation method. There was a highly significant effect of inoculation method $(P<0.001$ at 2 and 4 weeks), reflecting significantly greater incidence of brown spot in wounded $(96.3 \pm 1.7 \%)$ versus nonwounded $(31.5 \pm 5.1 \%)$ berries at 2 weeks, and likewise in wounded $(99.1 \pm 0.9 \%)$ versus nonwounded berries $(54.6 \pm 6.4 \%)$ at 4 weeks.

Conditional severity was evaluated based on the lesion diameter of infected berries 4 weeks after inoculation. There was a significant effect of both isolate $(P<0.001)$ and inoculation method $(P<0.001)$, with a significant interaction effect $(P=0.01)$. Therefore, isolate differences were examined separately for nonwound and wound inoculation, and the effect of inoculation method was examined separately for each isolate. There was no significant effect of isolate on lesion size following nonwound inoculation $(P=0.13)$ but the effect of isolate was significant when wound inoculated $(P<0.001)$, reflecting significantly smaller lesion diameter on $C$. cladosporioides-inoculated berries compared with berries inoculated by two $C$. herbarum species complex isolates (isolate $14=C$. limoniforme and isolate $38=$ C. tenellum), with intermediate lesion sizes for the other three C. herbarum species complex isolates (Table 3). The effect of inoculation method was significant for two $C$. herbarum species complex isolates (isolate $14=C$. limoniforme and isolate $38=C$. tenellum) $(P<0.001)$, reflecting greater lesion size following wound inoculation; there was no significant difference in lesion size of wounded and nonwounded berries for all other isolates (Table 3). In all trials, the identity of all Cladosporium isolates recovered from inoculated, symptomatic fruit corresponded to the inoculated isolate, and disease did not develop in negative controls.

Efficacy of epiphyte-targeted surface treatment strategies on postharvest disease development. There was no significant effect of surface disinfestation on brown spot incidence 2 weeks after treatment $(P=0.17)$, wherein 78 to $81 \%$ of clusters developed symptoms across both treatments (Table 4$)$. The effect of treatment on conditional severity 2 weeks after treatment was significant $(P=0.02)$, reflecting lower brown spot severity in the surface-disinfested $(17.8 \pm 2.2 \%$ of berries/ cluster) compared with the nondisinfested $(27.5 \pm 3.6 \%$ of berries/ cluster) treatment (Table 4).

\section{Discussion}

In this study, brown spot on $V$. vinifera Red Globe in the California central valley is described as a dry rot consisting of a dark brown to black spot on the fruit surface and a dark mycelial mat encasing the seed, which is often coated with olive-green spores. In some cases, internal growth occurs in the absence of an external spot, indicating that invasion of berry tissue may not necessarily originate from lesion formation on the fruit surface. This is supported by observations that Cladosporium spp. can establish asymptomatic infections within placental vascular tissue, possibly by entry through the stem rachis or through the ovary at bloom (C. L. Swett and W. D. Gubler, unpublished data), and by studies which have recovered Cladosporium spp. from internal tissues of asymptomatic berries (Dugan et al. 2002).

Table 2. Species-level identity of Cladosporium isolates recovered from brown spot symptoms, based on actin sequence homology with GenBank accessions of voucher specimens

\begin{tabular}{lccccc}
\hline Species & Complex $^{\mathbf{y}}$ & Voucher $^{\mathbf{z}}$ & GenBank accession & Brown spot isolates & Homology (bp) \\
\hline Cladosporium cladosporioides & $\mathrm{C}$ & $\mathrm{CBS} 112388^{\mathrm{T}}$ & $\mathrm{HM} 148490.1$ & 26 & $171 / 171$ \\
C. limoniforme & $\mathrm{H}$ & $\mathrm{CBS} 140484^{\mathrm{T}}$ & KT600592.1 & $1,2,3,4,5,14$ & $169 / 170$ \\
C. ramotenellum & $\mathrm{H}$ & $\mathrm{CBS} 121628^{\mathrm{T}}$ & EF679538.1 & 31,40 & $165 / 168(31) ; 168 / 168(40)$ \\
C. tenellum & $\mathrm{H}$ & $\mathrm{CBS} 121634^{\mathrm{T}}$ & $\mathrm{EF} 679555.1$ & 38 & $164 / 170$ \\
\hline
\end{tabular}

y $\mathrm{C}=$ cladosporioides species complex and $\mathrm{H}=$ herbarum species complex.

${ }^{\mathrm{z}} \mathrm{CBS}=$ Centraalbureau voor Schimmelcultures and superscript $\mathrm{T}$ indicates ex-type material 


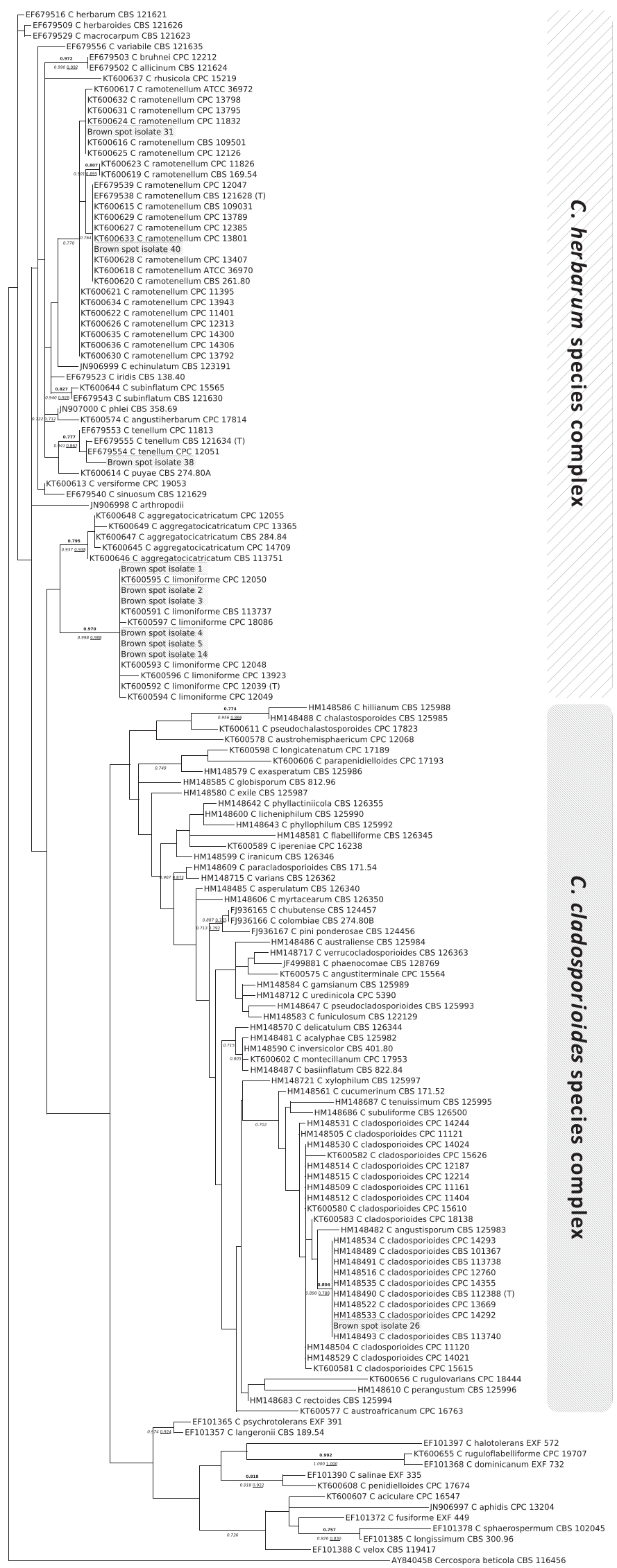

Fig. 2. Maximum likelihood tree inferred from partial sequences of the actin gene using the TPM $3 u f+l+G$ nucleotide substitution model. Support values of 0.700 and above from 1,000 bootstrap replicates are indicated on branches; on top in bold are values from this tree, with neighbor joining (italicized) and maximum parsimony (underlined) on the bottom. The branch to the Cercospora beticola outgroup was truncated for display purposes. Leaf names include GenBank accession numbers, species and strain names from Centraalbureau voor Schimmelcultures (CBS), American Type Culture Collection (ATCC), Culture Collection of Pedro Crous (CPC), and Culture Collection of Extremophilic Microorganisms (EXF; University of Ljubljana, Slovenia); (T) indicates accessions from ex-type material listed in Table 2. Figure produced with TreeGraph 2 (Stöver and Müller 2010) and LibreOffice Draw. 
To our knowledge, this is the first description of brown spot that includes formation of an internal mycelial structure. Preliminary studies indicate putative function as a heretofore unorganized survival structure for Cladosporium spp. (C. L. Swett and W. D. Gubler, unpublished data). Expansion of brown spot symptomology to include internal symptoms in the absence of a surface spot can aid in development of improved diagnosis guidelines to detect and eradicate infected berries prior to shipment, reducing costly losses associated with product breakdown during transport.

Disease surveys demonstrated that brown spot is not solely a postharvest disease but can also manifest in the field postveraison. In this region, brown spot has not previously been regarded as a preharvest disease; this may be due, in part, to the localization of symptoms on berries within the center of the cluster, which cannot be seen unless the cluster is dissected. The presence of brown spot in the field indicates that disease management should involve both pre- and postharvest practices. Further studies are needed to identify infection intervals in the field and evaluate efficacy of different preharvest treatments.

Analysis of relative frequency of association indicated that members of the $C$. herbarum species complex may function as the primary pathogens causing brown spot in Red Globe table grape in the central valley of California. In these studies, C. cladosporioides was rare and never recovered as the sole Cladosporium sp. from diseased berries. Moreover, $C$. cladosporioides was less virulent (smaller lesion diameter) than several $C$. herbarum species complex isolates. Analyses of $C$. herbarum isolates indicated segregation into three species, reflecting the rapidly changing face of Cladosporium; C. limonoforme was only recently described, and the range of habitats occupied by $C$. ramotenellum only recently expanded beyond Europe (Bensch et al. 2010, 2012, 2015; Schubert et al. 2007). However, phylogenetic groupings did not correlate with differences in biological function based on virulence assays, which indicated similar virulence across all $C$. herbarum species complex isolates. Perhaps more in-depth studies with a greater number of isolates per putative species and evaluation of additional biological traits may support segregation based on the ecological species concept. Additionally, multilocus analyses or analysis of more informative gene regions may better resolve Cladosporium community diversity.

All isolates were able to cause disease under cold-storage conditions, regardless of whether berries were mechanically wounded; however, wounding increased both disease incidence for all isolate treatments and disease severity for two isolates. These results indicate that epidermal wounding can increase losses from brown spot. Red Globe are typically heavily irrigated to market as an over-large table grape. In addition, grape berries are treated with sulfur and maintained at nearly $100 \% \mathrm{RH}$ during storage. These practices can result in alterations to the berry surface such as cuticle thinning, splitting, and hairline cracking that may facilitate colonization in the absence of macroscopic wounding (Zoffoli et al. 2011).

These results differ from previous studies, which identified C. cladosporioides as a dominate driver of brown spot, with members of the C. herbarum species complex occurring as a secondary colonists with weaker infection abilities (Briceño and Latorre 2008; Riggs 2006). However, our studies diverge from past analyses by examining disease development under cold-storage conditions in both species surveys and virulence assays. Perhaps, under colder temperatures, $C$. herbarum has greater growth, penetration, or enzymatic production capacities than C. cladosporioides. Studies of the effect of temperature on relative growth traits and virulence could provide important insights into niche differentiation and interactions within Cladosporium communities, and would have valuable translational implications for improving coldstorage and transport management.

Results from surface-treatment studies indicate that reduction of epiphytic populations can significantly reduce the severity of brown spot development under cold-storage conditions, providing support for the efficacy of current strategies targeting reduction of epiphytic populations leading up to and following harvest. However, there was no significant effect of surface treatment on brown spot incidence in the cluster, indicating that some infections are not controlled by targeting epiphytic populations late in the season. Although severity was lower in the short term of this study ( 2 weeks), surface treatments may lack efficacy over time periods sufficient for sporulation and secondary spread from diseased to healthy fruit.

Brown spot incidence and severity were both unexpectedly high in the surface-disinfested treatment ( $78 \%$ of clusters infected and $18 \%$ of berries infected/cluster). These results may reflect inefficacy of the surface disinfestation treatment or presence of preestablished (latent) infections in fruit at the time of treatment. It is possible that Cladosporium spp. primarily establish internal infections early in the season, during or following bloom, in a mode similar to B. cinerea (Coertze et al. 2001). In support of this hypothesis, preharvest surveys in this study indicate that these fungi can become established in fruit early in the season. In addition, preliminary studies indicate that up to $50 \%$ of asymptomatic Red Globe berries per cluster may sustain internal infections at the pea-size development stage (C. L. Swett and W. D. Gubler, unpublished data) and Cladosporium spp. have been recovered from asymptomatic wine grape berries in Washington state (Dugan et al. 2002). If Cladosporium spp. have a peak infection period in the spring or summer, preharvest

Table 4. Effect of cluster surface disinfestation at the time of harvest on brown spot development under cold-storage conditions

\begin{tabular}{lcc}
\hline Treatment & Incidence $(\%)^{\mathbf{y}}$ & Conditional severity $(\%)^{\mathbf{z}}$ \\
\hline Not disinfested & $81.3 \pm 7.8 \mathrm{a}$ & $27.5 \pm 3.6 \mathrm{a}$ \\
Surface disinfested & $78.1 \pm 7.3 \mathrm{a}$ & $17.8 \pm 2.2 \mathrm{~b}$ \\
\hline
\end{tabular}

${ }^{y}$ Brown spot incidence evaluated as percentage of clusters which developed brown spot 2 weeks post-treatment (two experiments combined). Means ( \pm standard error $[\mathrm{SE}]$ ) separated by different letters in the same column are significantly different, based on ANOVA $(P \leq 0.05)$.

${ }^{\mathrm{z}}$ Conditional severity of brown spot, quantified based on percentage of berries/cluster with brown spot symptoms 2 weeks posttreatment, in those clusters which developed symptoms (two experiments combined). Means $( \pm \mathrm{SE})$ separated by different letters in the same column are significantly different, based on ANOVA $(P \leq 0.05)$.

Table 3. Conditional severity of brown spot on Vitis vinifera Red Globe under cold-storage conditions, 4 weeks after inoculation

\begin{tabular}{|c|c|c|c|c|}
\hline \multirow[b]{2}{*}{ Species complex (SC), isolate } & \multirow[b]{2}{*}{ ID $^{y}$} & \multicolumn{2}{|c|}{ Lesion diameter $(\mathrm{mm})^{\mathrm{x}}$} & \multirow[b]{2}{*}{$P^{\mathbf{z}}$} \\
\hline & & Nonwound & Wound & \\
\hline \multicolumn{5}{|l|}{ Cladosporium herbarum SC } \\
\hline Brown spot isolate 31 & C. ramotenellum & $3.80 \pm 0.44 \mathrm{a}$ & $4.41 \pm 0.40 \mathrm{ab}$ & 0.338 \\
\hline Brown spot isolate 40 & C. ramotenellum & $4.30 \pm 0.65 \mathrm{a}$ & $5.87 \pm 0.56 \mathrm{~b}$ & 0.088 \\
\hline Brown spot isolate 38 & C. tenellum & $3.23 \pm 0.36 \mathrm{a}$ & $5.87 \pm 0.53 b$ & $<0.001$ \\
\hline Brown spot isolate 5 & C. limoniforme & $4.16 \pm 0.70 \mathrm{a}$ & $4.43 \pm 0.33 \mathrm{ab}$ & 0.696 \\
\hline Brown spot isolate 14 & C. limoniforme & $2.25 \pm 0.36 \mathrm{a}$ & $5.33 \pm 0.37 \mathrm{~b}$ & $<0.001$ \\
\hline \multicolumn{5}{|l|}{ C. cladosporioides SC } \\
\hline Brown spot isolate 26 & C. cladosporioides & $3.14 \pm 0.85 \mathrm{a}$ & $3.17 \pm 0.40 \mathrm{a}$ & 0.968 \\
\hline
\end{tabular}

${ }^{\mathrm{x}}$ Brown spot lesion size on those berries that developed symptoms (both experiments combined). Means ( \pm standard error) separated by different letters in the same column are significantly different, based on Tukey's multiple means comparison $(P \leq 0.05)$.

y Sequence-based ID. of Cladosporium spp. based on BLAST search and phylogenetic analyses.

${ }^{\mathrm{z}}$ Effect of inoculation treatment for each isolate, based on ANOVA. Significant difference denoted by $P \leq 0.05$. 
treatment strategies targeting early-season infections may be critical to reduce brown spot losses postharvest.

This study provides one of the first in-depth characterizations of the brown spot pathosystem in late-harvest table grape in California.

The discovery of additional brown spot symptoms that are restricted to internal tissues and analyses of brown spot impacts on fruit preharvest can be used to improve early detection and to minimize losses during storage and transport. Pathosystem characterization under cold-storage conditions provides new insight into the etiology and biology of this important disease, indicating that members of the C. herbarum species complex are primary causal agents, rather than C. cladosporioides. Efficacy of surface-treatment strategies provide support for current late-season management methods but also indicate that early-season control can reduce losses. Together, these studies can be used to inform management decisions and develop new strategies to control brown spot on table grape in California and other regions where table grape berries are grown for the late-season market.

\section{Acknowledgments}

We thank T. Harris for photo credit for Figure 1 and C. Young and K. Spawton for assistance in species isolation and identification.

\section{Literature Cited}

Altschul, S. F., Gish, W., Miller, W., Meyers, E. W., and Lipman, D. J. 1990. Basic Local Alignment Search Tool. J. Mol. Biol. 215:403-410.

Barnett, H. L., and Hunter, B. B. 1998. Illustrated Genera of Imperfect Fungi, 4th ed. American Phytopathological Society, St. Paul, MN.

Bensch, K., Braun, U., Groenewald, J. Z., and Crous, P. W. 2012. The genus Cladosporium. Stud. Mycol. 72:1-401.

Bensch, K., Groenewald, J. Z., Braun, U., Dijksterhuis, J., de Jesús YáñezMorales, M., and Crous, P. W. 2015. Common but different: The expanding realm of Cladosporium. Stud. Mycol. 82:23-74.

Bensch, K., Groenewald, J. Z., Dijksterhuis, J., Starink-Willemse, M., Andersen, B., Summerell, B. A., Shin, H. D., Dugan, F. M., Schroers, H. J., Braun, U., and Crous, P. W. 2010. Species and ecological diversity within the Cladosporium cladosporioides complex (Davidiellaceae, Capnodiales). Stud. Mycol. 67:1-94.

Briceño, E. X., and Latorre, B. A. 2007. Outbreaks of Cladosporium rot associated with delayed harvest wine grapes in Chile. Plant Dis. 91:1060.

Briceño, E. X., and Latorre, B. A. 2008. Characterization of Cladosporium rot in grapevines, a problem of growing importance in Chile. Plant Dis. 92:1635-1642.

Carbone, I., and Kohn, L. M. 1999. A method for designing primer sets for speciation studies in filamentous ascomycetes. Mycologia 91:553-556.

Castresana, J. 2000. Selection of conserved blocks from multiple alignments for their use in phylogenetic analysis. Mol. Biol. Evol. 17:540-552.

Coertze, S., Holz, G., and Sadie, A. 2001. Germination and establishment of infection on grape berries by single airborne conidia of Botrytis cinerea. Plant Dis. 85: 668-677.

Darriba, D., Taboada, G. L., Doallo, R., and Posada, D. 2012. jModelTest 2: More models, new heuristics and parallel computing. Nat. Methods 9:772.
Delp, C. J., Hewitt, W. B., and Nelson, K. E. 1951. Cladosporium rot of grapes in storage. Phytopathology 41:937-938.

Dugan, F. M., Lupien, S. L., and Grove, G. G. 2002. Incidence, aggressiveness and in planta interactions of Botrytis cinerea and other filamentous fungi quiescent in grape berries and dormant buds in Central Washington State. J. Phytopathol. 150:375-381.

Fernández-Trujillo, J. P., Obando-Ulloa, J. M., Baró, R., and Martínez, J. A. 2008. Quality of two table grape guard cultivars treated with single or dual-phase release $\mathrm{SO}_{2}$ generators. J. Appl. Bot. Food Qual. 82:1-8.

Green, P. 2015. Phrap, version 1.090518. Online publication. Laboratory of Phil Green, Genome Sciences Department, University of Washington. http://phrap.org

Green, P., and Ewing, B. 2015. Phred, version 0.071220c. Online publication. Laboratory of Phil Green, Genome Sciences Department, University of Washington. http://phrap.org/phredphrapconsed.html

Harvey, J. M., and Pentzer, W. T. 1960. Market Diseases of Grapes and Other Small Fruits. United States Department of Agriculture, Agricultural Marketing Service, Market Quality Research Division, Washington, DC.

Katoh, K., and Standley, D. M. 2013. MAFFT multiple sequence alignment software version 7: Improvements in performance and usability. Mol. Biol. Evol. 30:772-780.

Kumar, S., Stecher, G., and Tamura, K. 2016. MEGA7: Molecular Evolutionary Genetics Analysis version 7.0 for bigger datasets. Mol. Biol. Evol. 33:1870-1874

Larsson, A. 2014. AliView: A fast and lightweight alignment viewer and editor for large data sets. Bioinformatics 30:3276-3278.

Latorre, B. A., Briceño, E. X., and Torres, R. 2011. Increase in Cladosporium spp. populations and rot of wine grapes associated with leaf removal. Crop Prot. 30: $52-56$.

Maddison, D. R., and Maddison, W. P. 2014. Chromaseq: A Mesquite package for analyzing sequence chromatograms, version 1.12. Online publication. http:// mesquiteproject.org/packages/chromaseq

McRoberts, N., Hughes, G., and Madden, L. V. 2003. The theoretical basis and practical application of relationships between different disease intensity measurements in plants. Ann. Appl. Biol. 142:191-211.

Ouchi, S., Hatamoto, M., Oku, H., Shiraishi, T., Yokoyama, T., Tateishï, M., and Fujii, S. 1976. Brown spot of grapes caused by Cladosporium cladosporioides and Cladosporium herbarum. Sci. Rep. Fac. Agric. Okayama Univ. 48:17-22.

Riggs, P. 2006. Biology, epidemiology and control of Cladosporium cladosporioides causing Brown Spot on Vitis vinifera. Dissertation, University of California, Davis.

Rooney-Latham, S., Janousek, C. N., Eskalen, A., and Gubler, W. D. 2008. First report of Aspergillus carbonarius causing sour rot of table grapes (Vitis vinifera) in California. Plant Dis. 92:651.

Schubert, K., Groenewald, J. Z., Braun, U., Dijksterhuis, J., Starink, M., Hill, C. F., Zalar, P., De Hoog, G. S., and Crous, P. W. 2007. Biodiversity in the Cladosporium herbarum complex (Davidiellaceae, Capnodiales), with standardisation of methods for Cladosporium taxonomy and diagnostics. Stud. Mycol. 58:105-156.

Sprague, R. 1952. Field rots of grapes in north-central Washington. Northwest Sci. 27:1-16.

Stöver, B. C., and Müller, K. F. 2010. TreeGraph 2: Combining and visualizing evidence from different phylogenetic analyses. BMC Bioinf. 11:7.

Zoffoli, J. P., Latorre, B. A., and Yahia, E. M. 2011. Table grape (Vitis viniferd L.). Postharvest Biol. Technol. Trop. Subtrop. Fruits 3:179-212, 213e$214 \mathrm{e}$.

Zwickl, D. J. 2006. Genetic algorithm approaches for the phylogenetic analysis of large biological sequence datasets under the maximum likelihood criterion. Ph.D. dissertation, The University of Texas at Austin. 